Notification List

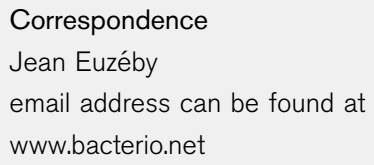

\section{Notification that new names and new combinations have appeared in volume 61 , part 2 , of the IJSEM}

This listing of names published in a previous issue of the IJSEM is provided as a service to bacteriology to assist in the recognition of new names and new combinations. This procedure was proposed by the Judicial Commission [Minute 11(ii), Int J Syst Bacterio/ 41 (1991), p. 185]. The names given herein are listed according to the Rules of priority (i.e. page number and order of valid publication of names in the original articles). Taxonomic opinions included in this List (i.e. the creation of synonyms or the emendation of circumscriptions) cannot be considered as validly published nor, in any other way, approved by the International Committee on Systematics of Prokaryotes and its Judicial Commission.

\begin{tabular}{|c|c|c|}
\hline Name/author(s): & Proposed as: & Page no. \\
\hline Yaniellaceae Li et al. 2008 pro synon. Micrococcaceae Pribram 1929 (Approved Lists 1980) & synon. $^{*}$ & 225 \\
\hline Auritidibacter Yassin et al. 2011 & gen. nov. & 228 \\
\hline Auritidibacter ignavus Yassin et al. 2011 & sp. nov. & 228 \\
\hline Micrococcineae Stackebrandt et al. 1997 emend. Yassin et al. 2011 & emend.* & 228 \\
\hline Haloarcula salaria Namwong et al. 2011 & sp. nov. & 234 \\
\hline Haloarcula tradensis Namwong et al. 2011 & sp. nov. & 235 \\
\hline Jeotgalicoccus coquinae Martin et al. 2011 & sp. nov. & 240 \\
\hline Jeotgalicoccus aerolatus Martin et al. 2011 & sp. nov. & 240 \\
\hline Aeromonas rivuli Figueras et al. 2011 & sp. nov. & 245 \\
\hline Burkholderia oxyphila Otsuka et al. 2011 & sp. nov. & 252 \\
\hline Comamonas zonglianii Yu et al. 2011 & sp. nov. & 257 \\
\hline Novosphingobium soli Kämpfer et al. 2011 & sp. nov. & 261 \\
\hline Allocatelliglobosispora Lee and Lee 2011 & gen. nov. & 268 \\
\hline Allocatelliglobosispora scoriae Lee and Lee 2011 & sp. nov. & 268 \\
\hline Parvibaculum indicum Lai et al. 2011 & sp. nov. & 273 \\
\hline Virgibacillus soli Kämpfer et al. 2011 & sp. nov. & 277 \\
\hline Paenibacillus chungangensis Park et al. 2011 & sp. nov. & 284 \\
\hline Kocuria salsicia Yun et al. 2011 & sp. nov. & 288 \\
\hline Hydrogenophilus islandicus Vésteinsdóttir et al. 2011 & sp. nov. & 294 \\
\hline Nitratireductor indicus Lai et al. 2011 & sp. nov. & 297 \\
\hline Saccharothrix variisporea corrig. (ex Tomita et al. 1977) Kim et al. 2011 & sp. nov., nom. rev. & 313 \\
\hline Photobacterium swingsii Gomez-Gil et al. 2011 & sp. nov. & 318 \\
\hline Micromonospora rhizosphaerae Wang et al. 2011 & sp. nov. & 323 \\
\hline Gangiinia Lee et al. 2011 & gen. nov. & 328 \\
\hline Gangjinia marincola Lee et al. 2011 & sp. nov. & 328 \\
\hline Formosa spongicola Yoon and Oh 2011 & sp. nov. & 332 \\
\hline Flavobacterium dongtanense Xiao et al. 2011 & sp. nov. & 345 \\
\hline Ruegeria marina Huo et al. 2011 & sp. nov. & 349 \\
\hline Pseudoalteromonas donghaensis $\mathrm{Oh}$ et al. 2011 & sp. nov. & 353 \\
\hline Arcobacter trophiarum De Smet et al. 2011 & sp. nov. & 360 \\
\hline Vibrio communis Chimetto et al. 2011 & sp. nov. & 365 \\
\hline Fontibacillus Saha et al. 2010 emend. Lee et al. 2011 & emend.* & 372 \\
\hline Fontibacillus panacisegetis Lee et al. 2011 & sp. nov. & 373 \\
\hline Oleibacter Teramoto et al. 2011 & gen. nov. & 378 \\
\hline Oleibacter marinus Teramoto et al. 2011 & sp. nov. & 378 \\
\hline Borrelia carolinensis Rudenko et al. 2011 & sp. nov. & 382 \\
\hline Undibacterium oligocarboniphilum Eder et al. 2011 & sp. nov. & 389 \\
\hline
\end{tabular}


cont.

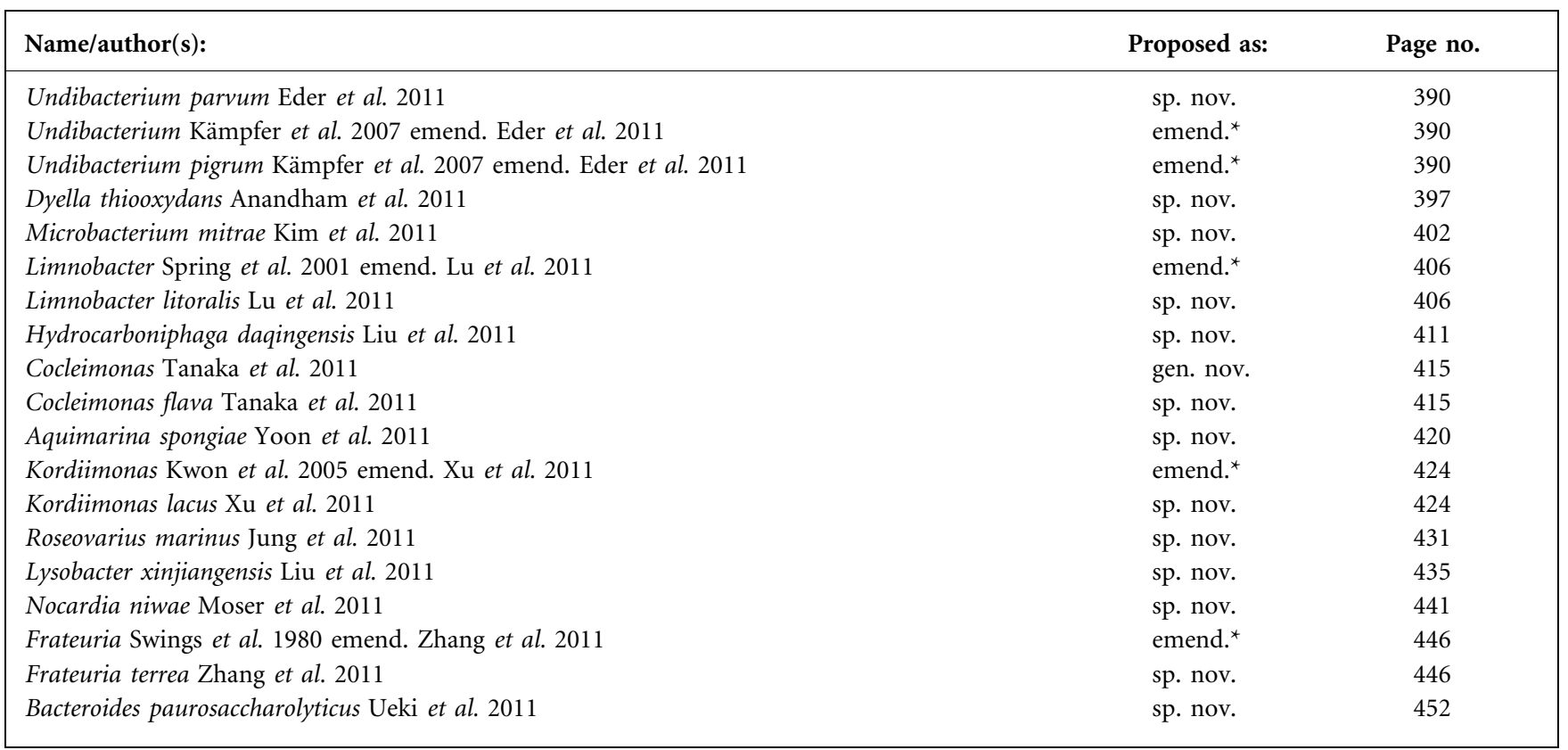

${ }^{\star}$ Taxonomic opinion. 